\title{
CERTAIN SUBGROUPS OF WEYL GROUPS ARE SPLIT
}

\author{
DANIEL GOLDSTEIN AND ROBERT M. GURALNICK
}

\begin{abstract}
Let $C$ be the centralizer in a finite Weyl group of an elementary abelian 2-subgroup. We show that every complex representation of $C$ can be realized over the field of rational numbers. The same holds for a Sylow 2-subgroup of $C$.
\end{abstract}

\section{Contents}

1. Introduction

2. Symmetric groups

3. Direct products

4. Wreath products

5. Division algebras

6. Schur indices

7. Classical Weyl groups

8. Exceptional Weyl groups

9. Positive characteristic

10. Real reflection groups

11. Open questions

References

Appendix A. Code

Appendix B. More code

\section{INTRODUCTION}

The purpose of this note is to generalize the well-known result that every complex representation of a finite Weyl group can be realized over the field of rational number $\mathbb{Q}$. Benard [2] proved this by a caseby-case analysis of the exceptional groups. Springer's cohomological construction [12, Corollary 1.5] gives a more uniform proof.

Date: November 16, 2018.

1991 Mathematics Subject Classification. 20C15, $20 \mathrm{G} 42$.

Key words and phrases. Weyl group, Schur indicator, Frobenius-Schur index, rationality of representations. 
We set some notation that is used throughout the paper. $W$ is a finite Weyl group, $E$ is an elementary abelian 2-subgroup of $W, C$ is the centralizer of $E$ in $W$, and $T$ is a Sylow 2-subgroup of $C$.

Theorem 1.1. All complex representations of the groups $C$ and $T$ can be realized over $\mathbb{Q}$.

Theorem 1.1 reduces to the result of Benard and Springer in the case $E=1$. The fact that every irreducible complex character of $C$ has values in $\mathbb{Q}$ is proved in [9]. The case of classical Weyl groups was treated in [7].

We say the finite group $G$ is split if each of its complex representations can be realized over $\mathbb{Q}$. With this terminology, Theorem 1.1 is the assertion that $C$ and $T$ are split. Clearly, $G$ is split if and only if each of its irreducible complex representations can be realized over $\mathbb{Q}$ (since every complex representation of $G$ is a direct sum of irreducible representations). Let $\operatorname{Irr}(G)$ denote the set of irreducible complex characters of $G$. We say that $\chi \in \operatorname{Irr}(G)$ is split if it is the trace of a $\mathbb{Q} G$-module. If $\chi$ is split, then $\chi$ is rational-valued, but the converse can fail (e.g. the quaternion group of order eight). In fact, $\chi$ is split if and only if $\chi$ is rational-valued and the Schur index of $\chi$ is equal to 1.

In this paper, we use both the Schur index and the Frobenius-Schur indicator. We remark that for a 2-group, the Schur index of an irreducible character is trivial if and only if the Schur index is +1 (Corollary 6.5).

We mention some easy reductions used in the proof. It suffices to prove the theorem for irreducible finite Weyl groups, as any finite Weyl group is a direct product of these (see Section 3).

An irreducible Weyl group is either of classical or exceptional type. The proof for a classical Weyl group $W$ is in Section 7 .

For the exceptional Weyl groups, we prove a slightly stronger statement (Theorem 8.1). We suspect (but do not prove) that this stronger statement also holds for the classical case.

Let $G$ be a finite group and $S$ a Sylow 2-subgroup of $G$. Then the following four conditions, if they hold, would imply that both $G$ and $S$ are split.

(1) Each irreducible complex character of $S$ is rational-valued.

(2) Each Frobenius-Schur indicator of $S$ is equal to +1 .

(3) Each irreducible complex character $\psi$ of $G$ occurs with odd multiplicity in an induced character from $S$.

(4) Each irreducible complex character $\psi$ of $G$ is rational-valued.

By the remark, the 2-group $S$ is split if and only if conditions (1) and (2) hold. 
Now assume that $S$ is a Sylow 2-subgroup with $S$ split.

Let $\psi$ be an irreducible complex character of $G$ such that conditions (3) and (4) hold. It follows from the theory of Schur indices and the Brauer-Speiser theorem (see [3], [11]) that $\psi$ is the trace of a $\mathbb{Q} G$ module (Corollary 6.3). Consequently, conditions (3) and (4), for all such $\psi$, would imply that $G$ is split.

We show that conditions (1)-(4) hold for every exceptional $(C, T)$ pair. We note that our proof of Theorem 1.1 for the classical groups does not use conditions (1)-(4). We do not know whether condition (3) holds for the classical groups.

Our proof of this stronger statement for an exceptional Weyl group $W$, relies heavily on a computer calculation. Our method is as follows. First, we enumerate the conjugacy classes of subgroups $C \leq W$ that are centralizers of an elementary abelian 2-subgroup. This list can be computed by an easy downward induction. (Choose an involution. Take its centralizer. Remove duplicate subgroups. Repeat. See Appendix A for Magma code [1] to do this.)

There is a good reason for using this sort of algorithm. Namely, the number of conjugacy classes of elementary 2-subgroups of $W$ can be much larger than the number of conjugacy classes of centralizers of elementary abelian 2-subgroups of $W$. For the symmetric groups, see Section 2 .

\section{SyMmetriC GROUPS}

Let $\mathrm{Sym}_{n}$ denote the symmetric group on $n$ letters. Let $e(n)$ denote the number of conjugacy classes of elementary abelian 2-subgroups of $\mathrm{Sym}_{n}$. Let $c(n)$ denote the number of conjugacy classes of centralizers of elementary abelian 2-subgroups of $\mathrm{Sym}_{n}$. In this section we show that $e(n) \geq c(n)$, at least for large $n$.

Let log denote the logarithm to the base 2 .

Lemma 2.1. Let $n \geq 1$. Then

(1) $\log c(n) \leq(\log n)^{2}$, and

(2) $\log e(n) \geq(n-3)^{2} / 16-n / 4-n \log n$.

Let $E$ be an elementary abelian 2-subgroup of $\mathrm{Sym}_{n}$. Each orbit of $E$ has size a power of 2 . Let $a_{k}$ be the number of orbits of size $2^{k}$. Let $C$ be the centralizer of $E$. Then $C$ is direct product of groups $C_{k}$, where each $C_{k}$ is a wreath product of the symmetric group $\operatorname{Sym}_{a_{k}}$ with an elementary abelian 2-group of order $2^{k}$ acting regularly on $2^{k}$ points.

It follows from this description that $c(n)$ is equal to the number of partitions of $n$ into powers of 2 . In other words, $c(n)$ is equal to the 
number of ways of writing

$$
n=a_{0} \cdot 1+a_{1} \cdot 2+a_{2} \cdot 4+\cdots+a_{k} \cdot 2^{k}
$$

with nonnegative integers $a_{0}, \ldots, a_{k}$.

Since $k \leq \log n$, and each $a_{i} \leq n$, it follows that $c(n) \leq n^{\log n}$.

Let $V$ be a vector space of dimension $2 m$ over the field of 2 elements. Then the number of subspaces of $V$ of dimension $m$ is

$$
\frac{\left(2^{2 m}-1\right)\left(2^{2 m}-2\right) \cdots\left(2^{2 m}-2^{m-1}\right)}{\left(2^{m}-1\right)\left(2^{m}-2\right) \cdots\left(2^{m}-2^{m-1}\right)} \geq \frac{2^{(2 m-1) m}}{2^{m^{2}}}=2^{m^{2}-m}
$$

Next we estimate the number of conjugacy classes of elementary abelian 2-subgroups of $\operatorname{Sym}_{n}$. Set $m=\lfloor n / 4\rfloor$. Let $E$ be an elementary abelian 2-group of rank $2 m$ acting on $4 m$ points, with $2 m$ orbits of size 2. By the previous paragraph, the number of subgroups of $E$ of order $2^{m}$ is at least $2^{m^{2}-m}$. Since each conjugacy class of subgroups can have at most $n$ ! members, we have $e(n) \geq 2^{m^{2}} /\left(2^{m} n !\right)$. Since $n ! \leq n^{n}$, we see that $\log e(n) \geq(n-3)^{2} / 16-n / 4-n \log n$.

This finishes the proof of the lemma. From the lemma and a direct calculation, one sees that $e(n) \geq c(n)$ for $n \geq 129$.

\section{Direct PRODUCTS}

By the following lemma, whose straightforward proof we omit, it suffices to prove Theorem 1.1 for irreducible finite Weyl groups.

Consider the property $P(G)$ of a group $G$ :

$P(G)$ : For each elementary abelian 2-subgroup $E$ of $G$, the centralizer of $E$ in $G$ and each of its Sylow 2subgroups are split.

Lemma 3.1. Let $A=\prod A_{i}$ be a finite direct product of finite groups. Then

(1) $A$ is split if and only if each $A_{i}$ is split.

(2) $P(A)$ holds if and only if $P\left(A_{i}\right)$ holds for each $i$.

\section{Wreath PRODUCTS}

Let $H$ be a finite group. Let $H_{n}$ denote the wreath product $H$ 2 $\mathrm{Sym}_{n}$. We show that for each $n>0, H$ is split if and only if $H_{n}$ is split.

First we construct some $\mathbb{Q} H_{n}$-modules. Given a $\mathbb{Q} H$-module $M$, let $\operatorname{Sym}_{n}$ act on the $n$-fold tensor product $M^{\otimes n}$ by permuting coordinates. As this action is compatible with the $\mathbb{Q} H^{n}$-module structure, $M^{\otimes n}$ acquires a $\mathbb{Q} H_{n}$-module structure. 
Finally, for any $\mathbb{Q} H$-module $M$ and any $\mathbb{Q} \operatorname{Sym}_{n}$-module $N$, we define the $\mathbb{Q} H_{n}$-module $W(M, N)=M^{\otimes n} \otimes N$. On the first factor, $H_{n}$ acts as described in the previous paragraph. On the second factor, $H_{n}$ acts through its quotient $\operatorname{Sym}_{n}=H_{n} / H^{n}$.

Its dimension is $\operatorname{dim} W(M, N)=(\operatorname{dim} M)^{n} \cdot \operatorname{dim} N$. If $M$ and $N$ are irreducible, then $W(M, N)$ is irreducible.

Let $M_{1}, \ldots, M_{k}$, be representatives for the isomorphism classes of irreducible $\mathbb{Q} H$-modules. Set $d_{i}=\operatorname{dim} M_{i}$.

Let $\mathcal{A}$ denote the set of tuples $a=\left(a_{1}, \ldots, a_{k}\right)$ of nonnegative integers with sum $a_{1}+\cdots+a_{k}=n$. For $a=\left(a_{1}, \ldots, a_{k}\right) \in \mathcal{A}$, set $P_{a}=$ $\prod_{1 \leq i \leq k} \operatorname{Sym}_{a_{i}}$. Then

$$
\left|\operatorname{Sym}_{n}: P_{a}\right|=\frac{n !}{a_{1} ! \cdots a_{k} !}
$$

For each $a \in \mathcal{A}$, let $\mathcal{N}_{a}$ denote the set of irreducible $\mathbb{Q} P_{a}$-modules. $\left(\mathcal{N}_{a}\right.$ may be identified (via tensor product) with the set of tuples $\left(N_{1}, \ldots, N_{k}\right)$, where each $N_{i}$ is an irreducible $\mathbb{Q}$ Sym $_{a_{i}}$-module).

For $a \in \mathcal{A}$ and $\nu \in \mathcal{N}_{a}$, form the $H_{n} . P_{a}$-module

$$
\bigotimes_{1 \leq i \leq k} W\left(M_{i}, N_{i}\right)
$$

and let $Z(a, \nu)$ be the $H_{n}$-module obtained by induction. The $\mathbb{Q} H_{n^{-}}$ modules $Z(a, \nu)$ are irreducible and pairwise nonisomorphic. We have

$$
\begin{aligned}
\operatorname{dim} W(a, \nu) & =\left|\operatorname{Sym}_{n}: P_{a}\right| \operatorname{dim} \nu, \text { and } \\
\sum_{\nu \in N_{a}}(\operatorname{dim} \nu)^{2} & =\left|P_{a}\right|
\end{aligned}
$$

Now we have all the ingredients necessary to prove our result on wreath products.

Theorem 4.1. Let $H$ be a finite group. Let $n \geq 1$ be an integer. Then $H$ is split if and only if $H_{n}=H 2 \mathrm{Sym}_{n}$ is split.

Proof. In one direction, assume that $H_{n}$ is split and let $V$ be an irreducible $\mathbb{Q} H$-module. Form the irreducible $\mathbb{Q} H_{n}$-module $W(V$, triv $)=$ $\mathrm{V}^{\otimes \mathrm{n}}$. Since $W(V$, triv) is absolutely irreducible, so is $V$. 
Conversely, suppose that $H$ is split. Enumerate as above the irreducible $\mathbb{Q} H$-modules. We have

$$
\begin{aligned}
\sum_{a} \sum_{\nu \in N_{a}} \operatorname{dim} Z(a, \nu)^{2} & =\sum_{a} \sum_{\nu}\left(\left|\operatorname{Sym}_{n}: P_{a}\right| \operatorname{dim} \nu\right)^{2} \\
& =\sum_{a}\left|\operatorname{Sym}_{n}: P_{a}\right|^{2} \sum_{\nu}(\operatorname{dim} \nu)^{2} \\
& =\left|\operatorname{Sym}_{n}\right| \sum_{a}\left|\operatorname{Sym}_{n}: P_{a}\right| \\
& =n ! \sum_{a} \frac{n !}{a_{1} ! \cdots a_{k} !} \\
& =n ! k^{n}
\end{aligned}
$$

where the last equality follows from the multinomial formula. Since $n ! k^{n}=\left|H_{n}\right|$, we see that every irreducible complex representations of $H_{n}$ is equal to one of the $Z(z, \nu)$. Since these are all split, the theorem is proved.

Corollary 4.2. Let $G$ be a finite group that is split. Let $m \geq 1$ be a power of 2, S a Sylow 2-subgroup of $\mathrm{Sym}_{m}$. Let $G$ < $S$ be the wreath product given by the permutation action coming from $S \leq \mathrm{Sym}_{m}$. Then $G$ ㄴ $S$ is split.

Proof. Set $m=2^{r}$. Then $r \geq 0$. If $r=0$ there is nothing to show. If $r=1$ then $\operatorname{Sym}_{m}$ is a 2-group, so the result follows from Theorem 4.1. We proceed by induction on $r>1$. Then, $S=T$ ? $(\mathbb{Z} / 2 \mathbb{Z})$, where $T$ is a Sylow 2-subgroup of $\operatorname{Sym}_{2^{r-1}}$. Thus $G<S \cong(G<T)<(\mathbb{Z} / 2 \mathbb{Z})$. The result follows by induction and the case $r=1$.

\section{Division ALGEBRAS}

We record some known results about division algebras that are needed for Section 6 on Schur indices.

Let $K$ be field. The collection $\operatorname{Br}(K)$ of all (isomorphism classes of) division algebras that are finite-dimensional and central over $K$ has the structure of additive group. The identity element is $K$ itself and the inverse of $D$ is $D^{\text {opp }}$, the opposite algebra. This group law can be characterized be the rule $D+D^{\prime}=D^{\prime \prime}$ whenever

$$
D \otimes D^{\prime} \cong \operatorname{Mat}_{n}\left(D^{\prime \prime}\right)
$$

for some $n$. (Such a $D^{\prime \prime}$ and an $n$ always exist.)

If $L$ is an extension of $K$, then there is a map $e_{L / K}: \operatorname{Br}(K) \rightarrow \operatorname{Br}(L)$ characterized by the property: $e_{L / K}(D)=D^{\prime}$ if $D \otimes_{K} L=M_{n}\left(D^{\prime}\right)$ for some $n$. (Such a $D^{\prime}$ and an $n$ always exist.) 
Suppose $K$ is a finite extension of $\mathbb{Q}$. Let $\mathcal{M}$ denote the set of places of $K$, including the infinite places. Let $K_{v}$ denote the completion of $K$ at the place $v \in \mathcal{M}$. Let $k$ be one of the $K_{v}$ 's.

It is known that $\operatorname{Br}(k)=\mathbb{Q} / \mathbb{Z}$ if $k$ is non-Archimedean, $\operatorname{Br}(k)=$ $\frac{1}{2} \mathbb{Z} / \mathbb{Z}$ if $k$ is the real field, and $\operatorname{Br}(k)=\{0\}$ if $k$ is the complex field.

Let $D$ be a finite-dimensional division algebra with center $K$. The local-global theory of division algebras over a number field assigns to each place $v$ in $\mathcal{M}$ a local invariant $\operatorname{inv}_{v}(D) \in \operatorname{Br}\left(K_{v}\right)$.

The local invariants enjoy the following properties.

(1) For each $D, \operatorname{inv}_{v}(D)$ is nonzero for at most finitely many $v \in \mathcal{M}$.

(2) For each $D$, the sum $\sum_{v \in \mathcal{M}} \operatorname{inv}_{v}(D)$, which is well-defined by (1), is equal to 0 .

(3) Let $\left(\epsilon_{v}\right)_{v \in \mathcal{M}}$ be any collection of local invariants $\epsilon_{v} \in \operatorname{Br}\left(K_{v}\right)$ that satisfy (1) and (2). Then there exists a $D$ such that $\operatorname{inv}_{v}(D)=\epsilon_{v}$ for all $v \in \mathcal{M}$.

(4) If $\epsilon_{v}(D)=\epsilon_{v}\left(D^{\prime}\right)$ for all $v \in \mathcal{M}$, then $D$ is isomorphic to $D^{\prime}$.

Suppose $|L: K|<\infty$. The local invariants of $D$ and of $e_{L / K}(D)$ are related as follows. Let $w$ be a place of $L$ lying over the place $v$ of $K$, and let $L_{w}$ denote the completion of $L$ at $w$. Then $\operatorname{inv}_{w}\left(e_{L / K}(D)\right)=$ $\left|L_{w}: K_{v}\right| \operatorname{inv}_{v}(D)$.

Let now $D$ be a finite-dimensional division algebra $D$ with center $F$, $F$ an arbitrary field. There exists a maximal commutative subalgebra $A$ of $D$. The dimension of such an $A$ is called the degree of $D$. This does not depend on the choice of $A$, since $\left(\operatorname{dim}_{F} A\right)^{2}=\operatorname{dim}_{F} D$. The rank of $F$ itself is 1 , and a division algebra of rank 2 is a quaternion algebra.

Lemma 5.1. Let $D$ be a finite-dimensional division algebra with center $K$, a finite extension of $\mathbb{Q}$. Then $D \cong D^{\text {opp }}$ if and only if $D$ has order $\leq 2$ in the Brauer group $\operatorname{Br}(K)$. If this condition holds, then $D$ is a quaternion algebra or $D=K$.

Proof. The first statement follows from the definition of inversion in the Brauer group. The condition holds if and only if each local invariant is equal to 0 or $1 / 2$. Let $\left(\epsilon_{v}\right)_{v \in \mathcal{M}}$ be a collection of local invariants $\epsilon_{v} \in \operatorname{Br}\left(K_{v}\right)$ that satisfy (1) and (2) and such that for all $v, \epsilon_{v} \in \frac{1}{2} \mathbb{Z} / \mathbb{Z}$. We may assume that not all the local invariants are zero.

Let $S$ be the set of places of $K$ such that $\epsilon_{v}$ is nonzero. By (1) and (2), $S$ is a finite set of even cardinality, and if $v \in S$ then $K_{v}$ is not the complex field. By weak approximation, there is $x$ in $K$ whose image in $K_{v}$ is a nonsquare for each $v \in S$. Let $L=K(\sqrt{x})$. Then $\left|L_{w}: K_{v}\right|=2$ for each $v \in S$, where $L_{w}$ is the completion of $L$ at a place $w$ lying 
over $v$. It follows that all the local invariants of $D \otimes_{K} L$ are trivial. By (3) applied to $L$, we see that $D \otimes_{K} L \cong M_{n}(L)$ for some $n$. If $L$ is a splitting field for $D$, then $\operatorname{dim} D \leq|L: K|^{2}$.

In the case $K=\mathbb{Q}$ one can be much more explicit. Now let $\mathcal{M}$ be the set of places of $\mathbb{Q}$. Let $\left(\epsilon_{v}\right)_{v \in \mathcal{M}}$ be a collection of local invariants $\epsilon_{v} \in \frac{1}{2} \mathbb{Z} / \mathbb{Z}$, at most finitely many of which are nonzero, such that $\sum_{v \in \mathcal{M}} \epsilon_{v}=0$. One can show directly that a quaternion algebra over $\mathbb{Q}$ exists with local invariants $\left(\epsilon_{v}\right)$ by appealing to Dirichlet's theorem.

For $a, b$ nonzero elements of $\mathbb{Q}$, one constructs the quaternion algebra $(a, b)$. Its local invariant at the place $v$ is easily calculated using the Hilbert symbol

$$
\epsilon_{v}((a, b))=\langle a, b\rangle_{v}
$$

A good reference for the Hilbert symbol is [10, Chapter III].

Let $S$ be the set of places $v$ of $\mathbb{Q}$ such that $\epsilon_{v}$ is nonzero. We assume $S$ contains 2 and the infinite place, and leave the other cases, which are treated similarly, to the reader.

Set $T=S \backslash\{2, \infty\}$. Choose for each prime $p \in T$ a nonsquare $a_{p}$ in $\mathbb{Z} / p \mathbb{Z}$. By Dirichlet's theorem and the Chinese remainder theorem, there exists a positive prime number $q$ such that

$$
\begin{aligned}
q & \equiv-a_{p}(\bmod p) \quad \text { for all } p \in T, \text { and } \\
q & \equiv 3 \quad(\bmod 8) .
\end{aligned}
$$

Let $t=\prod_{p \in T} p$. Now the quaternion algebra $D^{\prime}=(-2 t,-q)$ does the trick. We have $\operatorname{inv}_{\infty}\left(D^{\prime}\right)=\langle-2 t,-q\rangle_{\infty}$ is nonzero, since $\left.t, q\right\rangle 0$. For $p \in T$, we have $\operatorname{inv}_{p}\left(D^{\prime}\right)=\left\langle-2 t, a_{p}\right\rangle=1 / 2$. At the prime 2 , we have $\operatorname{inv}_{2}\left(D^{\prime}\right)=\langle-2 t,-q\rangle_{2}=\langle-2 t, 5\rangle_{2}=\langle 2,5\rangle_{2}\langle-t, 5\rangle_{2}=1 / 2$. Clearly, $\operatorname{inv}_{v}\left(D^{\prime}\right)=0$ for places $v$ not in $S \cup\{q\}$. We have just seen that $D^{\prime}$ has invariant $1 / 2$ at each place $v \in S$. Therefore, since the sum of the local invariants is 0 , and $|S|$ is even, it follows that $\operatorname{inv}_{q}\left(D^{\prime}\right)=0$.

Finally, it follows by (4) that $D^{\prime} \cong D$ for any division algebra $D$ with local invariants $\operatorname{inv}_{v}(D)=\epsilon_{v}$.

\section{SCHUR INDICES}

The relation to Schur indices is as follows. Let $\chi$ be an irreducible complex character of the finite group $G$. Let $K$ be an extension of $\mathbb{Q}$ that contains the values of $\chi$.

The Schur index of $\chi$ over $K$ is the least positive integer $m_{\chi}$ such that $m_{\chi} \chi$ is the trace of a $K G$-module. By definition, $m_{\chi}=1$ if and only if $\chi$ itself is afforded by a $K G$-module. We observe that for any 
positive integer $n, n \chi$ is the trace of a $K G$-module if and only if $n$ is a multiple of $m_{\chi}$.

It is clear that a $K G$-module of trace $m_{\chi} \chi$ is irreducible. Let $M$ be a $K G$-module of trace $m_{\chi} \chi$. By Schur's Lemma, $D=\operatorname{End}_{K}(M)$ is a division algebra. The Schur index $m_{\chi}$ is the degree of $D$.

Lemma 6.1. (Brauer [3], Speiser [11]) If $\chi$ is real-valued, then $m_{\chi} \leq 2$.

Proof. (Fein [4]). Let $K$ be a totally real number field containing the values of $\chi$. Let $m=m_{\chi}$.

Let $V$ be an irreducible $K G$-module whose trace is $m \chi$. Set $D=$ $\operatorname{End}_{K G}(V)$. We are required to show that the degree of $D$ over $K$ is at most 2. In view of Lemma 5.1, it suffices to show $D$ is isomorphic to its opposite.

However, this follows easily from the fact that $\chi$ is real-valued. Let $V^{\prime}=\operatorname{Hom}_{K}(V, K)$ be the vector space dual to $V$. Endow $V^{\prime}$ with a $G$-action by declaring $(g \cdot \lambda)(v)=g^{-1}(\lambda v)$. The character of $V^{\prime}$ is $m \bar{\chi}=m \chi$ since $\chi$ is real-valued. Clearly, $\operatorname{End}_{K G}\left(V^{\prime}\right)$ is isomorphic to the opposite algebra to $D$, and consequently $D \simeq \operatorname{End}_{K G}(V) \simeq$ $\operatorname{End}_{K G}\left(V^{\prime}\right) \simeq D^{\text {opp }}$.

Lemma 6.2. Let $\chi$ be an irreducible complex character of the finite group $G$. Let $K$ be an extension of $\mathbb{Q}$ that contains the values of $\chi$. If $\rho$ is a character of a KG-module, then $m_{\chi} \mid(\chi, \rho)$.

Proof. Let $M$ be a $K G$-module affording the character $\rho$. The $\chi$ isotypic part of $M$ is defined over $K$. The lemma follows from the observation.

Corollary 6.3. Let $H$ be a subgroup of the finite group $G$. Let $\chi \in$ $\operatorname{Irr}(G)$ be rational-valued. Let $\rho$ be a character of $H$ corresponding to a representation defined over $\mathbb{Q}$. If the integer $\left(\rho_{H}^{G}, \chi\right)$ is odd, then $\chi$ is the trace of a representation defined over $\mathbb{Q}$.

Proof. This is a consequence of Lemmas 6.1 and 6.2.

Let $K$ be a finite extension of $\mathbb{Q}$ that contains the values of $\chi$. Let $\mathcal{M}$ be the set of places of $K$, including the infinite places. Since the completion $K_{v}$ contains $K$, hence a priori the values of $\chi$, it makes sense to compare the Schur index of $\chi$ over $K$ with the local Schur index of $\chi$ over $K_{v}$.

We record some basic facts. See [5, Corollary 9.5] for a proof of part (2).

Lemma 6.4. Let $\chi$ be an irreducible complex character of the finite group $G$. 
(1) The Schur index $m_{\chi}=1$ if and only if each of the local Schur indices is equal to one. Somewhat more is true. Let $v_{0} \in \mathcal{M}$. If the local Schur index is one at each place $v \neq v_{0}$ then so also is the Schur index at $v_{0}$ and the global Schur index.

(2) If $p$ is a prime and $p$ does not divide the order of $G$, then the p-adic Schur index is 1.

(3) The relation to the Frobenius-Schur indicator is as follows. Let $K$ be the field generated over $\mathbb{Q}$ by the values of $\chi$, and let $k$ be the completion of $K$ at an infinite place. The FrobeniusSchur indicator is 0 if $k=\mathbb{C}$. The Frobenius-Schur indicator is +1 (resp. -1 ) if $k=\mathbb{R}$ and the local Schur index at $\mathbb{R}$ is 1 (resp. 2).

Lemma 6.5. Let $S$ be a 2-group. Suppose that $\chi$ in $\operatorname{Irr}(S)$ has rational values and Frobenius-Schur indicator +1 . Then there is a $\mathbb{Q} S$-module with trace $\chi$.

Proof. Since $\chi$ is real-valued, the local Schur index of $\chi$ at the real place is 1. By Lemma 6.4(2), for all odd primes $p$, the local Schur index at $p$ is 1 . Hence, by Lemma 6.4(1) with $v_{0}=2$, we see that $m_{\chi}=1$. It follows that $\chi$ is afforded by a $K S$-module, where $K$ is the field generated over $\mathbb{Q}$ by the values of $\chi$. But by hypothesis, $K=\mathbb{Q}$.

We also need the following:

Lemma 6.6. Let $N$ be a subgroup of index 2 in the finite group $G$. Let $V$ be an irreducible $\mathbb{C} N$-module with character $\chi$. Let $W$ be an irreducible constituent of $V_{N}^{G}$. If $\chi$ takes values in $K$ and $W$ is defined over $K$, then $V$ is defined over $K$.

Proof. Let $M=V_{N}^{G}$ be the induced module. Either $M$ is irreducible or a sum of two distinct irreducible modules. In the former case, $M=W$, and in the latter case, $M$ is the sum of $W$ and a twist of $W$. In particular, since $W$ is defined over $K$, so also is $M$.

If $M$ is not irreducible, then $\left.M\right|_{N} \cong V$ is defined over $K$ by assumption. If $M$ is irreducible, then $\left(\chi_{N}^{G}, \chi_{N}^{G}\right)=1$, and so, by Frobenius reciprocity and by Lemma $6.2, m_{\chi}$ divides $\left(\chi,\left.\left(\chi_{N}^{G}\right)\right|_{N}\right)=1$. Since the trace of $V$ takes values in $K$, it follows via Lemma 6.6 that $V$ is defined over $K$.

\section{Classical Weyl groups}

We remark that there are many proofs that the symmetric group $\mathrm{Sym}_{n}$ is split. For one such see [8]. 
Let $G$ be a finite group. We note that all of the irreducible complex characters of $G$ are rational-valued if and only if, for all $g \in G$ and all integers $r$ coprime to $|G|$, there exists $y \in G$ such that $g^{r}=g^{y}$. Similarly, all of the irreducible complex characters of $G$ are real-valued if and only if $g$ is conjugate to $g^{-1}$ for all $g \in G$. We will need the following lemma.

Lemma 7.1. Let $G$ be a finite group all of whose irreducible complex characters are rational-valued. Let $G_{0}<G$ be a subgroup of index 2 . Let $g \in G$, and let $r$ be an integer coprime to $|G|$. If $G_{0}$ does not contain the centralizer in $G$ of $g$, then $g^{r}=g^{y}$ for some $y \in G_{0}$.

Proof. Since all of the irreducible complex characters are rational-valued, there exists $y$ in $G$ such that $g^{r}=g^{y}$. By hypothesis, there exists $u \in G \backslash G_{0}$ that centralizes $g$. Then both $y$ and $u y$ conjugate $g$ to $g^{r}$, and precisely one of these lies in $G_{0}$.

For $X$ a group, let $X \imath(\mathbb{Z} / 2 \mathbb{Z})$ denote the group generated by $X \times X$ and its automorphism $\tau(x, y)=(y, x)$.

Lemma 7.2. Let $X$ be a finite group with all representations defined over $\mathbb{Q}$.

(1) The group $Y=X \imath(\mathbb{Z} / 2 \mathbb{Z})$ is split.

(2) Let $X_{0}<X$ be a subgroup of index 2, and suppose that $X_{0}$ is split. Let $W_{0} \leq X \times X$ be the subgroup consisting of those pairs $(x, y)$ such that $x y \in X_{0}$. Then $W=\left\langle W_{0}, \tau\right\rangle$ is split.

Proof. Statement (1) is the assertion of Theorem 4.1 for $K=\mathbb{Q}$ and $n=2$, and we turn to (2).

We claim that all irreducible complex characters of $W_{0}$ are rationalvalued. Indeed, this is the case for $X_{0} \times X_{0}$, and if $w \in W_{0} \backslash\left(X_{0} \times X_{0}\right)$, then the centralizer in $X \times X$ of $w$ is not contained in $W_{0}$, and applying Lemma 7.1 to $W_{0} \leq X \times X$ proves the claim.

Note that $W /\left(\bar{X}_{0} \times X_{0}\right)$ is the Klein four group, hence $W$ is the union of three proper subgroups of index 2 , namely, $W_{0}, X_{0}$ ? $(\mathbb{Z} / 2 \mathbb{Z})$, and a subgroup conjugate to $X_{0} 2(\mathbb{Z} / 2 \mathbb{Z})$ in $Y$. Since each of these three subgroups has all irreducible complex characters rational-valued, the same is true for $W$, by the well-known criterion: all irreducible complex characters of the finite group $G$ are rational-valued if and only if for all $g \in G$, and all integers $r$ coprime to $|G|, g \sim g^{r}$.

Part (2) now follows from Lemma 6.6 applied to the index-2 inclusion $W \leq Y$. 
Lemma 7.3. Let $E$ be an elementary abelian 2-subgroup of $\mathrm{Sym}_{n}$. Let $C$ be the centralizer of $E$ in $\mathrm{Sym}_{n}$, and let $S$ be a Sylow 2-subgroup of $C$.

(1) $C$ is split.

(2) If $E$ has no fixed points, then $C \cap \mathrm{Alt}_{n}$ is split.

(3) $S$ is split.

(4) $S \cap \mathrm{Alt}_{n}$ is split.

Proof. Let $\Omega$ denote the set of cardinality $n$ on which $\operatorname{Sym}_{n}$ acts.

We prove (1) and (3) together. If $n=1$, then $C=S=1$ is split. We proceed by induction on $n$.

Assume that $E$ does not act regularly on some $E$-orbit $\Omega$. Then there is a partition $\Omega=\Omega_{1} \coprod \Omega_{2}$, such that the kernel of the action of $E$ on $\Omega_{1}$ has no fixed points on $\Omega_{2}$. Thus each $\Omega_{i}$ is invariant under the centralizer $C$ of $E$. Let $C_{i}$ be the centralizer in $\operatorname{Sym}\left(\Omega_{i}\right)$ of the image of $E$. Then $C=C_{1} \times C_{2}$ and $S=S_{1} \times S_{2}$, where $S_{i}$ is the Sylow 2-subgroup of the centralizer of an elementary abelian 2-group in a smaller symmetric group. Now $C$ and $S$ are split by the inductive hypothesis and Lemma 3.1.

Suppose $E$ acts regularly on all $E$-orbits. Then $C=E$ 々 Sym $_{d}$ where $n=d|E|$ and so $C$ is split by Theorem 4.1.

Similarly, if $S$ is not transitive on $\Omega$, then we can write $\Omega=\Omega_{1} \coprod \Omega_{2}$, where each $\Omega_{i}$ is $S$-invariant. As above, (3) follows by induction.

Suppose $S$ is transitive on $\Omega$. Then $S=E 々 S_{0}$ where $S_{0}$ is a Sylow 2-subgroup of a symmetric group of degree $m$, where $m|E|=|\Omega|$. Necessarily $m$ is a power of 2 . Now $S$ is split by Corollary 4.2 applied to $E=G$. This finishes the proof of (1) and (3) and we turn now to $(2)$.

Assume $E$ has no fixed points, and set $D=C \cap$ Alt $_{n}$. We claim that if the integer $r$ is coprime to the order of $g \in C$, then there exists $d \in D$ such that $g^{d}=g^{r}$. Clearly, the claim, if true, would imply (2) by Lemma 7.1 .

As above, we are done by induction if some orbit of $E$ is not regular. So we assume that is the case. Note that if $|E| \geq 4$, then $C \leq \operatorname{Alt}_{n}$ and the claim follows from (1).

Assume $E$ of order 2 acts semiregularly. The nonidentity element of $E$ then is a fixed-point-free involution. Any $c \in C$ can be written

$$
c=\sigma\left(e_{1}, \ldots e_{m}\right)
$$

where $2 m=n, e_{i} \in E$, and $\sigma \in \operatorname{Sym}_{m}$. If $\sigma$ is not a $m$-cycle, the claim follows by induction.

If $e_{1} e_{2} \cdots e_{m} \neq 1$, then $\sigma \notin D$ and the claim follows from Lemma $7.2(1)$. 
If $e_{1} e_{2} \cdots e_{m}=1$, then $c$ is conjugate to $\sigma$. The claim now follows as $\sigma$ and $\sigma^{r}$ are conjugate in $\operatorname{Sym}_{m} \leq$ Alt $_{n}$. This finishes the proof of the claim and of (2).

We prove (4). Let $T=S \cap \operatorname{Alt}_{n}$. Since $S$ is split and $|S: T|=2$, it suffices to show that all irreducible complex characters of $T$ are rationalvalued. We will prove the equivalent property: if $s \in S$ and $r$ is odd, then $s^{r}=s^{g}$ for some $g \in T$.

As above, if $S$ is not transitive, the result follows by induction. If $S$ is transitive, then all orbits of $E$ are regular. Lemma $7.2(2)$ shows that $T$ is split.

Our main result for the classical Weyl groups now follows.

Theorem 7.4. Let $E$ be an elementary abelian subgroup of a classical Weyl group $W$. Let $C$ be the centralizer of $E$ in $W$, and $T$ a Sylow 2-subgroup of $C$. Then both $C$ and $T$ are split.

Proof. For $W$ of type $A_{n}$, this is equivalent to assertions (1) and (3) of Lemma 7.3. The case of type $B_{n}$ is a special case of the type $A_{2 n}$ result. (Indeed, since $W$ of type $B_{n}$ is the centralizer of a fixed point free involution $z$ in $\mathrm{Sym}_{2 n}$, it follows that the centralizer in $W$ of $E$ equals the centralizer in $\mathrm{Sym}_{2 n}$ of the elementary abelian 2-subgroup $\langle z, E\rangle$.) For $W$ of type $D_{n}, n$ odd, this follows from Lemma 3.1, since $W \times(\mathbb{Z} / 2 \mathbb{Z})$ is a Weyl group of type $B_{n}$. For $W$ of type $D_{n}, n$ even, this follows from Lemma 7.3(2) and (4) since $W=B_{n} \cap \operatorname{Alt}_{2 n}$.

\section{Exceptional Weyl groups}

For the exceptional Weyl groups, we prove somewhat more than Theorem 1.1.

Theorem 8.1. Let $W$ be the Weyl group of an exceptional complex simple Lie algebra $\left(G_{2}, F_{4}, E_{6}, E_{7}\right.$, or $\left.E_{8}\right)$. Let $C$ be the centralizer of an elementary abelian 2-subgroup of $W$. Let $T$ be a Sylow 2-subgroup of $C$.

(1) All characters of $T$ are rational-valued. All Frobenius-Schur indicators of $T$ are equal to +1 . Consequently, $T$ is split.

(2) For every $\chi \in \operatorname{Irr}(C)$, there exists $\psi \in \operatorname{Irr}(T)$ such that $\left(\chi, \psi_{T}^{C}\right)$ is odd.

(3) All irreducible characters of $C$ are rational-valued.

The proof of Theorem 8.1 is a direct calculation in Magma [1] (see [6, Appendix A]). Now Theorem 1.1 follows from Theorem 8.1, the BrauerSpeiser Theorem and Lemma 6.2. 
We have already seen that part (1) of Theorem 8.1 holds for the classical Weyl groups. We have verified part (2) for $\mathrm{Sym}_{n}, 1 \leq n \leq 25$ by a direct calculation in Magma (see [6. Appendix B]), and we we suspect that it holds for all classical Weyl groups.

\section{Positive CHARACTERISTiC}

In order to apply our result to representations in positive characteristic, we appeal to a result of Thompson [13, p. 327]. Let $G$ be a finite group, $F$ an algebraically closed field of positive characteristic $p$, and $V$ an irreducible FG-module. Thompson's result states that if $p$ is odd and $V$ is self-dual, then there is an irreducible self-dual characteristic zero representation $W$ of $G$, such that $V$ appears with odd multiplicity as a composition factor of the reduction $\bmod p$ of $W$. Moreover, any such self-dual $W$ has the same Frobenius-Schur indicator as that of $V$.

Corollary 9.1 (Corollary to Thompson's result). Let $G$ be a finite group all of whose irreducible complex representations can be defined over $\mathbb{Q}$. Let $F$ be an algebraically closed field of positive characteristic $p$. Then every irreducible FG-module $V$ is self-dual and can be defined over the prime field. Furthermore, if $p$ is odd, then the Frobenius-Schur indicator of $V$ is +1 .

Proof. Since $G$ is split, all characters of $G$ are real-valued, and so every element of $G$ is conjugate to its inverse. It follows that $V$ is self-dual.

Since $g^{r} \sim g$ for $r$ coprime to $|G|$, it follows that the trace of $g$ on $V$ has values in the prime field. It now follows from Wedderburn's theorem that $V$ can be defined over the prime field.

Suppose $p$ is odd, and let $W$ be a characteristic zero irreducible module such that $V$ occurs in the reduction of $W$ modulo $p$ with odd multiplicity (such a $W$ exists by Thompson's result). Since $G$ is split, $W$ has Frobenius-Schur indicator +1 , whence $V$ has Frobenius-Schur indicator +1 , as was to be shown.

As a consequence of Corollary 9.1 and Theorem 1.1, we have:

Corollary 9.2. Let $W$ be a Weyl group and $E$ an elementary abelian 2-subgroup of $W$. Let $C$ be the centralizer of $E$ in $W$, and let $T$ be a Sylow 2-subgroup of $C$. Let $R=C$ or $T$. If $F$ is an algebraically closed field of odd characteristic, then every irreducible F $R$-module is self-dual, can be realized over the prime field, and has Frobenius-Schur indicator +1 . 


\section{REAL REFLECTION GROUPS}

A transformation $g \in G L_{n}(\mathbb{C})$ is a real reflection if $\operatorname{dim} \operatorname{ker}(g-$ $\left.I_{n}\right)=n-1$ and $\operatorname{dim} \operatorname{ker}\left(g+I_{n}\right)=1$. A finite subgroup of $G L_{n}(\mathbb{C})$ that is generated by real reflections is a real reflection group. This class includes the finite Weyl groups. In the context of this paper, it is natural to ask which real reflection groups are split.

Since every real reflection group is a direct product of irreducible real reflection groups, and in view of Theorem 3, we will only consider irreducible reflection groups.

The irreducible real reflection groups that are not Weyl groups are $H_{3}, H_{4}$ and the dihedral groups $I_{n}$ of order $2 n$ for $n=5$ and $n \geq 7$.

Let $G=H_{3}$ or $H_{4}$. Then $G$ is not split. The centralizer of a noncentral elementary abelian 2-subgroup of $G$ is split. The centralizer of any elementary abelian 2-subgroup of $G$ has a Sylow 2-subgroup that is split.

The group $I_{n}$ is split if and only if $n \leq 4$. A non-central elementary abelian 2-subgroup is self-centralizing, has order 4 and is split. A Sylow 2 -subgroup of $I_{n}$ is split if and only if $n$ is not divisible by 8 .

\section{Open questions}

We proved Theorem 8.1(2) for the exceptional Weyl groups only.

Question 11.1. Does the conclusion of Theorem 8.1(2) hold for the classical Weyl groups?

Question 11.2. Is it true that a Sylow 2-subgroup of a split finite group is split?

Let $G$ denote an arbitrary finite group and $S$ a Sylow 2-subgroup of $G$. As we have observed, a 2-group is split if and only if all characters have values in $\mathbb{Q}$ and all Frobenius-Schur indicators are +1 .

Thus, the previous question divides naturally into two parts.

Question 11.3. If all irreducible characters of $G$ have Frobenius-Schur indicators +1 , is the same true for $S$ ?

Question 11.4. If all irreducible characters of $G$ are rational-valued is the same true for $S$ ?

We also ask:

Question 11.5. If all irreducible characters of $G$ have Frobenius-Schur indicator equal to +1 , and all irreducible characters of $S$ are real-valued, then do all irreducible characters of $S$ have Frobenius-Schur indicator equal to +1 ? 


\section{REFERENCES}

[1] W. Bosma, J. Cannon, and C. Playoust, The Magma algebra system 1: the user language, J. Symbolic Comput. 24 (1997), 235-265.

[2] M. Benard, On the Schur indices of characters of the exceptional Weyl groups, Ann. of Math., 94 (1971), 89-107.

[3] R. Brauer, Über Zusammenhange zwischen arithmetischen und invariententheoretischen Eigenschaften von Gruppen linearer Substitutionen, Sitzber. Preuss. Akad. Wiss. (1926), 410-416.

[4] B. Fein, A note on the Brauer-Speiser theorem, Proc. Amer. Math. Soc. 25 (1970), 620-621.

[5] W. Feit. The Representation Theory of Finite Groups. North-Holland Mathematical Library Vol. 25, North-Holland (1982).

[6] D. Goldstein and R. Guralnick, Certain subgroups of Weyl groups are split, Arxiv.

[7] R. Guralnick and S. Montgomery, Frobenius-Schur indicators for subgroups and the Drinfel'd double of Weyl groups, Trans. Amer. Math. Soc. 361 (2009), no. 7, 3611-3632.

[8] G. James, The Representation Theory of the Symmetric Groups, Lecture Notes in Math. 682, Springer (1978).

[9] S. Kolesnikov, On the rationality and strong reality of Sylow 2-subgroups of Weyl and alternating groups, Algebra Logic 44 (2005), 25-30.

[10] J.-P. Serre, A Course in Arithmetic, Springer (1973).

[11] A. Speiser, Zahlentheoretische Sätze aus der Gruppentheorie, Math. Z. 5 (1919), 1-6.

[12] T. Springer, A construction of representations of Weyl groups, Invent. Math, 44 (1978), 279-293.

[13] J. Thompson, Some finite groups which appear as $\operatorname{Gal}(L / K)$, where $K \subseteq$ $\mathbb{Q}\left(\mu_{n}\right)$, in Lecture Notes in Math. 1185 (1986), 210-230. Lecture 6: Bilinear forms in characteristic $p$ and the Frobenius-Schur indicator.

\section{Appendix A. Code}

QQ := Rationals();

// Definition. The complex representation of the finite

// group X is *split* if it can be defined over QQ.

// $\mathrm{X}$ is *split* if all of its (irreducible) complex

// representations are split. A necessary condition

// for X to be split is that it has *rational character

// values*, i.e. if all its character values lie in QQ.

//

// Note that the converse holds if $\mathrm{X}$ is a 2-group.

// Given the character table ct of a finite group $g$, // "blah(ct)" returns TRUE if all character values of 
// $\mathrm{g}$ are rational and if all the Frobenius-Schur

// indicators are 1, and FALSE otherwise.

/////////////////////////////////////////////

function blah(ct)

b1 := \&and [ct $[i][j]$ in $Q Q: i, j$ in [1..\#ct]];

b2 $:=$ \&and $[\operatorname{Schur}(j, 2)$ eq 1: $j$ in $c t]$;

return $\mathrm{b} 1$ and $\mathrm{b} 2$;

end function;

// On input subsets $A$ and $B$

// of a finite group $g$, such

// that no two elements of

// $\mathrm{A}$ are conjugate in $\mathrm{g}$,

// "dedupe $(\mathrm{g}, \mathrm{A}, \mathrm{B})$ " outputs a

// maximal subsetC of AUB such

// that no two elements of $\mathrm{C}$

$/ /$ are conjugate in $\mathrm{g}$.

$/ / / / / / / / / / / / / / / / / / / / / / / / / / /$

function dedupe $(\mathrm{g}, \mathrm{A}, \mathrm{B})$

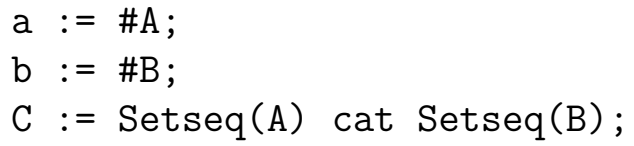

flag : $=[C[j+a]$ notin $A: j$ in $[1 \ldots b]]$;

for $i$ in $[1 \ldots a+b], j$ in $[1 \ldots b]$ do

if $i$ lt $j+a$ and $f l a g[j]$ and IsConjugate $(g, C[i], C[j+a])$ then $f \operatorname{lag}[j]:=$ false;

end if;

end for;

return $\{C[j+a]: j$ in $[1 \ldots b] \mid f l a g[j]\}$;

end function;

// On input a finite group $\mathrm{X}$,

// "bs(X)" tries to show that $X$ is split using

// the Brauer-Speiser theorem

// 1. Suppose X is a 2-group. Outputs TRUE if X has

// rational character values and FALSE otherwise.

// 2. Suppose $X$ is not a 2-group. Let $s$ be a Sylow

// 2-subgroup of $\mathrm{X}$.

// a. Construct all (classes of)

// irreducible representations of $\mathrm{s}$.

// b. Induce these up to $X$, to get a set $I$ of

// split reps of $X$. Try to prove $X$ split 


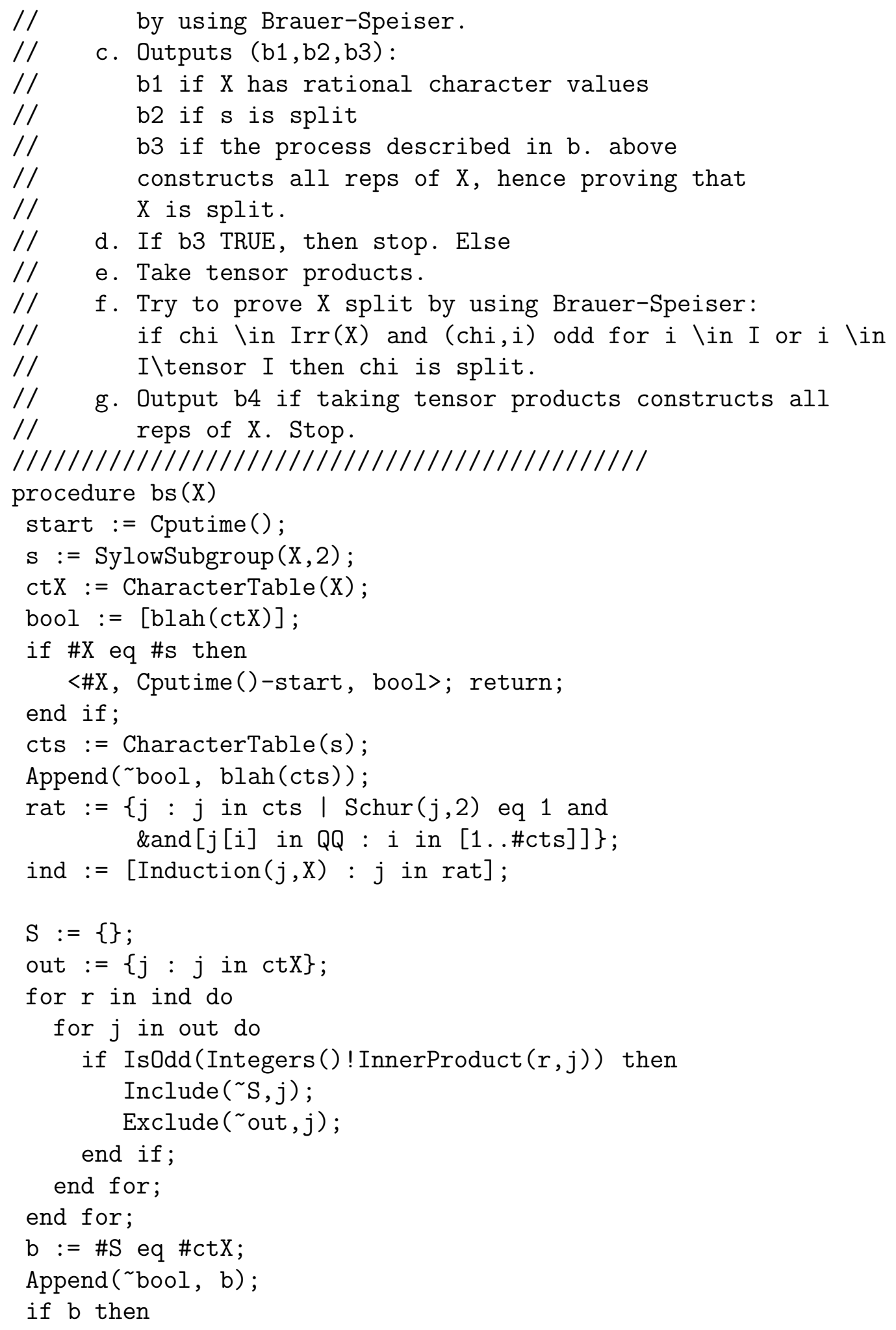




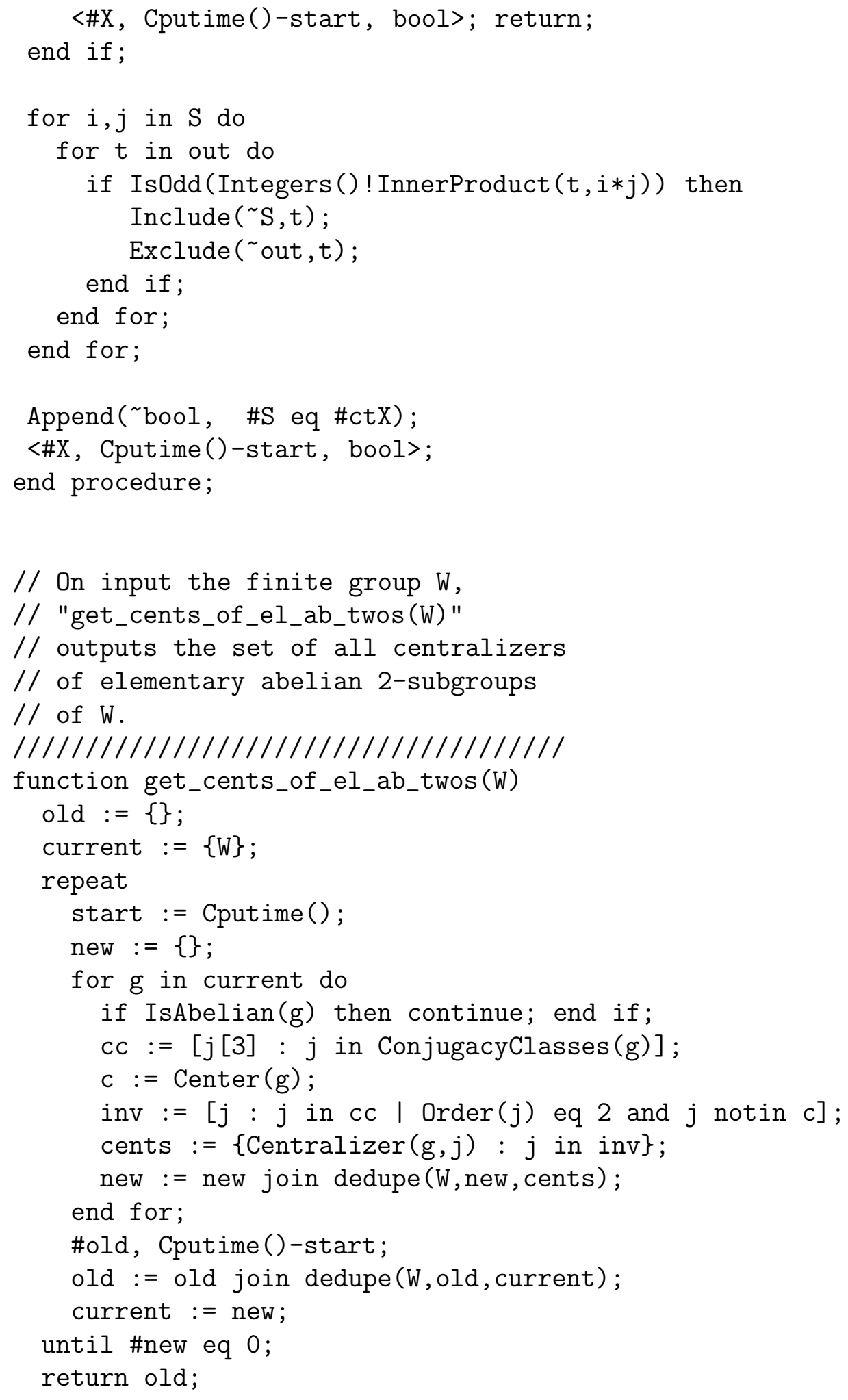




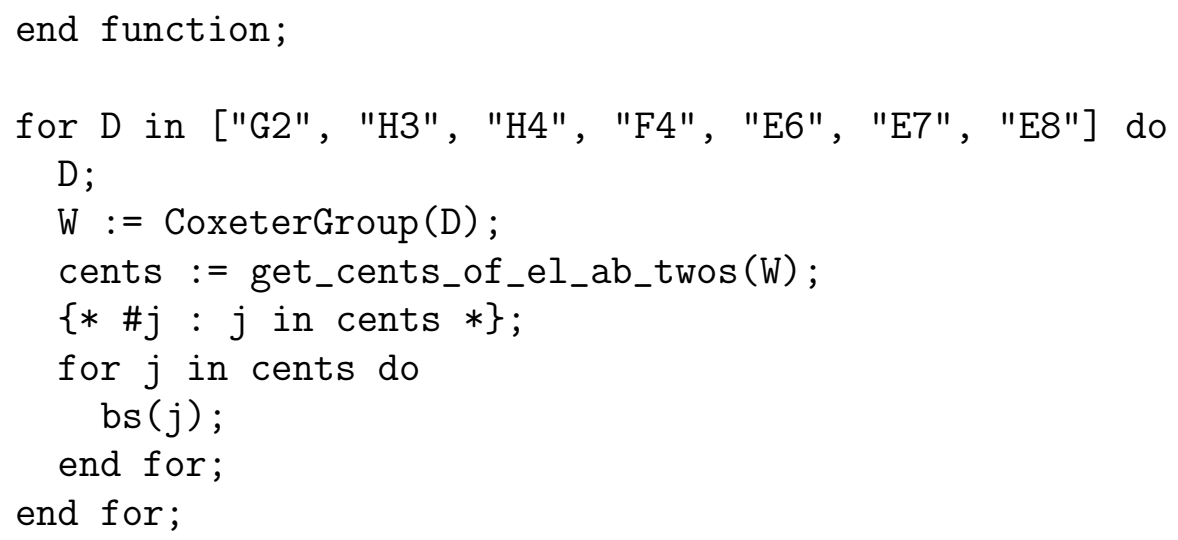

Appendix B. More CODE

// On input $\mathrm{n}$, "sym(n)"

// constructs the matrix

// with rows $\operatorname{Irr}\left(\mathrm{S}_{-} \mathrm{n}\right)$

$/ /$ and columns $\operatorname{Irr}(\mathrm{s})$,

// for s a Sylow 2-subgroup,

$/ /$ end entries the mod 2

// inner product with the

// induced character.

$/ / / / / / / / / / / / / / / / / / / / / / / / / / / / / / / /$

function $\operatorname{sym}(\mathrm{n})$

$\mathrm{Pa}:=$ Partitions ( $\mathrm{n})$;

charsg := [SymmetricCharacter $(\mathrm{pa}):$ pa in $\mathrm{Pa}$ ]

$\mathrm{g}:=\operatorname{Sym}(\mathrm{n})$;

$\mathrm{s}:=\operatorname{SylowSubgroup}(\mathrm{g}, 2)$;

cts := CharacterTable $(\mathrm{s})$;

res := [Restriction $(j, s): j$ in charsg];

return Matrix([[Integers()! InnerProduct(j, $x) \bmod 2$ :

end function; $j$ in cts]: $x$ in res]);

// On input a matrix $M$

// "oneineachrow(M)"

// outputs TRUE if there is at

// least one 1 in each row,

$/ /$ and FALSE otherwise.

$/ / / / / / / / / / / / / / / / / / / / / / / / / / / / / / / / /$

function oneineachrow $(\mathrm{M})$;

c : $=\operatorname{Ncols}(\mathrm{M})$; 


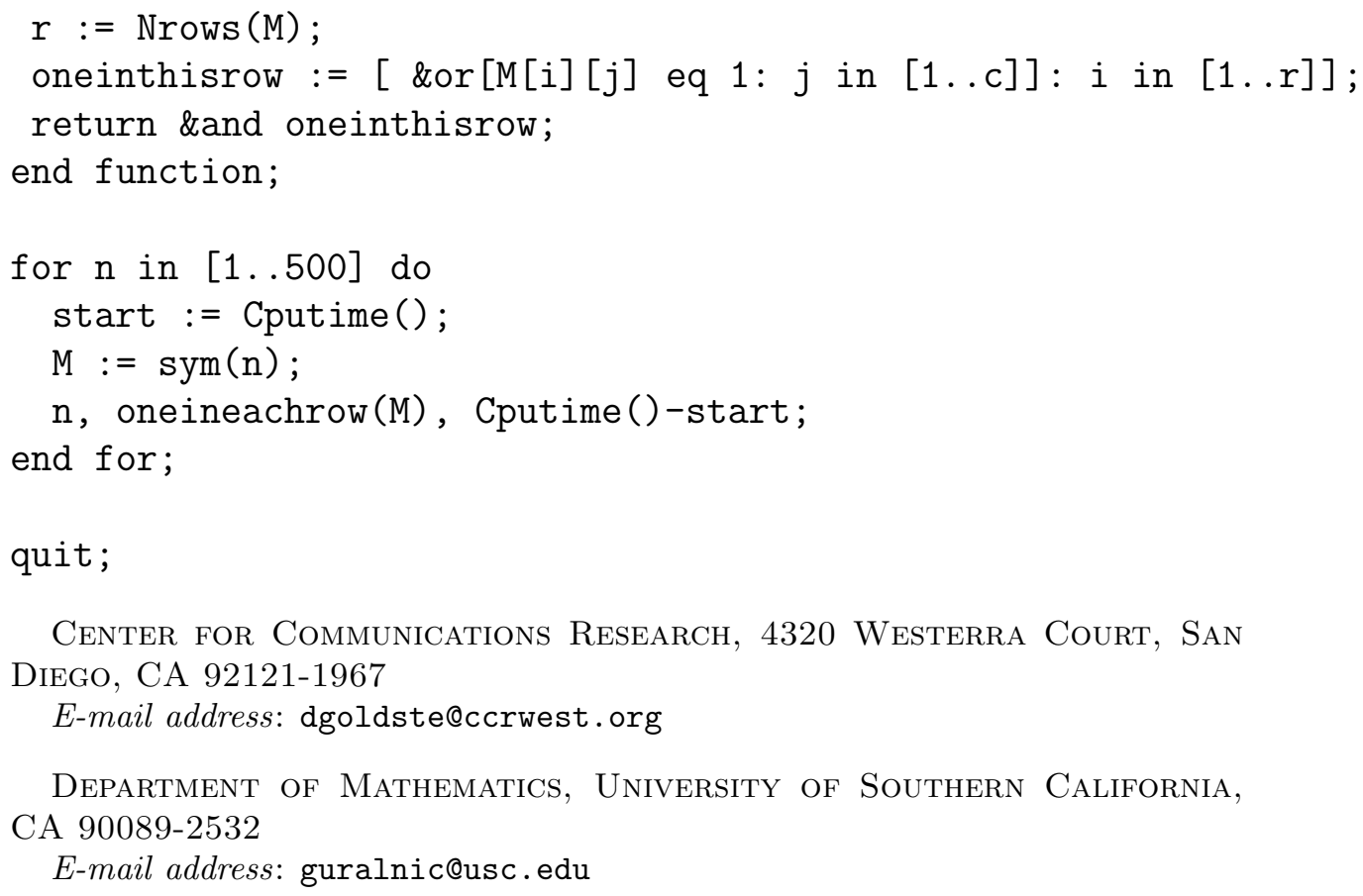

